\title{
Social and Economic Political Ideology Consistently Operate as Mutual Suppressors: Implications for Personality, Social, and Political Psychology
}

\author{
Thomas H. Costello* \\ Emory University, Department of Psychology \\ Scott O. Lilienfeld \\ Emory University, Department of Psychology \\ University of Melbourne, School of Psychological Sciences
}

NOTE: This manuscript has been accepted for publication in its current form in Social Psychological and Personality Science. This version is not the copy of record and may not exactly replicate the final, authoritative version of the article.

Preprint first posted: 06/08/2020

Last updated: 09/11/2020

*Corresponding author: Thomas H. Costello, 36 Eagle Row, Department of Psychology, Emory University, Atlanta, GA, 30322. E-mail: thcoste@emory.edu. 


\begin{abstract}
Right-left political views can be decomposed into distinct economic and social dimensions that bear differing relations with external criteria. In three community samples (total $\mathrm{N}=1487$ ), we identified replicable suppressor situations in which statistically controlling for either social or economic political ideology increased the other ideology dimension's relations with variables reflecting cognitive rigidity, authoritarianism, dangerous worldview, and lethal partisanship. Specifically, positive relations between social conservatism and these outcomes were enhanced after controlling for economic conservatism, while, after controlling for social conservatism, positive bivariate relations between economic conservatism and external criteria became negative and negative bivariate relations were enhanced. We identified similar, albeit less consistent, suppressor phenomena for general personality. Taken together, our results suggest that social and economic conservatism differ substantially in their psychological implications, and that following statistical control these differences emerge in samples in which social and economic conservatism are highly positively correlated.
\end{abstract}

Keywords: political psychology, suppression, social and economic conservatism, authoritarianism, personality. 


\section{Social and Economic Ideology Consistently Operate as Mutual Suppressors: Implications for Personality, Social, and Political Psychology}

In many nations around the world, political discourse is organized around an overarching left/liberal vs. right/conservative ideological dimension (Jost et al., 2009). This dimension traces its roots to the early stages of the French Revolution, with right vs. left reflecting preferences for stability (right; i.e., the aristocracy, seeking to preserve France's hereditary monarchy) vs. change (left; i.e., individuals advocating for a new regime). The ideals of "Liberté, égalité, fraternité" and opposition to authority, hierarchy, and tradition soon came to typify the political left. Over time, however, these distinctions have evolved, reflecting a broad swath of ideals and worldviews that often manifest idiosyncratically, or even contradictorily, across cultures and contexts (Caprara \& Vecchione, 2018).

Scholars have long sought to understand the psychological characteristics that predict individuals' willingness to embrace broad-based left- or right-wing political beliefs (Adorno et al., 1950). One longstanding research tradition, for instance, argues that right-wing policy preferences accord with needs for certainty and safety and, as such, coalesce to form a conservative "syndrome" (Hetherington \& Weiler, 2018; Jost et al., 2003). Relatedly, Jost and colleagues (2013, p. 1) argued that all manner of conservative ideologies explain the world in a manner that justifies the legitimacy of the prevailing sociocultural system, and preferences for such system justification are the "glue" that holds both left- and right-wing ideologies together.

Nevertheless, increasing evidence calls into question whether the right-left dimension adequately captures the complexity of individual differences in the political domain (Duckitt \& Sibley, 2009; Lameris et al., 2018; Malka et al., 2017; Pan \& Xu, 2018). Right-left political views can be decomposed into factorially distinct economic and social content subdimensions 
that may bear differing and sometimes opposing relations with personality traits and cognitive styles (Johnston \& Ollerenshaw, 2020). Social conservatism chiefly concerns traditional values, cultural rules, and norms, and is consistently positively related to certainty- and safety-related variables (Van Hiel et al., 2016). In contrast, economic conservatism, which chiefly concerns opposition to redistributive and regulatory economic interventions, has sometimes manifested null or even negative relations with certainty- and safety-related variables, including authoritarianism (Cizmar et al., 2014), threat sensitivity (Hibbing et al., 2014), needs for closure and structure (Van Hiel et al., 2004; Yilmaz \& Saribay, 2016), and reversed levels of (a) need for cognition (Feldman \& Johnston, 2014), (b) openness to experience (Carney et al., 2008), and (c) cognitive ability (Carl, 2014).

Such evidence highlights the possibility that right-wing social attitudes and left-wing economic attitudes fulfill similar needs. Just as maintaining tradition fosters a sense of certainty and safety, the promise of an economic "safety net" may mitigate uncertainties tied to living in a free-market economy. Relatedly, some authors have suggested that motivations to sacrifice personal freedoms for governmental protection provide the psychological link between left-wing economic policies and right-wing social policies (Malka et al., 2017). In contrast, some studies suggest roughly equivalent relationships between social and economic conservatism, on the one hand, and external criteria, on the other (Azevedo et al., 2019; Cornelis \& Van Hiel, 2006; Everett, 2013; Sterling et al., 2016), especially in politically engaged samples. Thus, whether and to what extent social and economic ideology have common psychological underpinnings remains undetermined (Federico, 2020).

One complicating factor that may partially account for these mixed findings is that bottom-up processes, such as psychological needs, are not the sole drivers of political ideology. 
Top-down processes, such as socialization, expressive partisanship, group membership, and partisan sorting, may also play critical roles (Converse, 1964; Kalmoe, 2020). Such top-down influences may lead large portions of the public, including people who are dispositionally inclined towards right-wing social policies and left-wing economic policies (or vice versa), to be tribalistically inclined to self-identify as liberal (or conservative) on both social and economic issues, thereby artificially inflating the correlation between social and economic ideology. In other words, participants' partisan identification with the global terms "conservative" or "liberal," rather than their dispositional tendency to support conservative or liberal policies per se, may introduce statistical noise that renders the unique elements of social and economic ideology more difficult for researchers to discern (Malka \& Lelkes, 2010; Morris et al., 2008).

Accordingly, statistically controlling for shared variance between social and economic ideology may serve to "clear the brush," clarifying social-economic divergences by minimizing respondent error stemming from top-down determinants of political affiliation. More specifically, social and economic ideology may be associated with suppressor effects, in which statistically controlling for one dimension increases (rather than decreases, as is observed in more typical cases of statistical redundancy) the magnitude of the other dimension's relation with relevant external criteria (Nickerson, 2008; Paulhus et al., 2004). As observed by Watson et al. (2013), suppressor effects often help to bring "into clearer focus opposing elements that are inherent—but largely hidden—in the measure's overall score" (p. 929), because they frequently result from the removal of nonspecific shared variance.

Replicable suppressor phenomena have traditionally been considered rare in psychology (e.g., Wiggins, 1973). Nevertheless, growing evidence suggests that the strategy of mutually controlling for complementary constructs is helpful for disentangling overlapping constructs, 
such as self-esteem and narcissism (Orth et al., 2016; Paulhus et al., 2004), shame and guilt (Orth et. al, 2006; Paulhus et al., 2004; Tracy \& Robins, 2006), authentic and hubristic pride (Tracy et al., 2009), collective narcissism and positive in-group identification (de Zavala et al., 2013), and adaptive and maladaptive dimensions of psychopathic personality (Lilienfeld et al., 2018). In the present studies, we investigate the hypothesis that the potentially divergent correlates of social and economic conservatism will be similarly clarified when the two constructs are mutually controlled for one another (i.e., differential relations between social and economic ideology will become larger after mutual control).

We generally expect to find mutual suppression, which occurs when two positively correlated predictors show larger regression weights after they are included in the same regression (Nickerson, 2008). Specifically, in cases where social and economic conservatism both manifest positive (or negative) bivariate regression coefficients with a given outcome, we expect to find mutual net suppression (Cohen \& Cohen, 1975; Conger, 1974; Nickerson, 2008; Paulhus et al., 2004), an instance of mutual suppression that occurs when one of the two partial regression coefficients shows a sign opposite to its corresponding bivariate coefficient. Nonmutual net suppression, which is net suppression where one of the two partial coefficients does not account for more variance than its corresponding bivariate coefficient (Nickerson, 2008), is also plausible and would be consistent with our expectations. Moreover, in cases where social and economic conservatism manifest bivariate regression coefficients with opposite signs, we expect to find cooperative suppression, such that controlling for their overlap boosts both regression weights. Table 1 presents examples of each type of suppression. 
Table 1. Examples of suppression situations.

\begin{tabular}{|c|c|c|c|c|c|c|c|}
\hline \multirow[b]{2}{*}{$\begin{array}{c}\text { Step } 1 \\
\text { Predictor }\end{array}$} & \multirow[b]{2}{*}{$\begin{array}{c}\text { Step } 2 \\
\text { Predictor }\end{array}$} & \multicolumn{2}{|c|}{$\begin{array}{c}\text { Step 1 (Bivariate } \\
\text { Coefficient) }\end{array}$} & \multicolumn{2}{|c|}{$\begin{array}{c}\text { Step } 2 \text { (Partial } \\
\text { Coefficients) }\end{array}$} & \multirow[b]{2}{*}{$\begin{array}{c}\text { Type of } \\
\text { Suppression }\end{array}$} & \multirow[b]{2}{*}{$\begin{array}{c}\text { Mutual } \\
\text { Suppression? }\end{array}$} \\
\hline & & Social $\beta$ & Economic $\beta$ & Social $\beta$ & Economic $\beta$ & & \\
\hline $\begin{array}{c}\text { Economic } \\
\text { Social }\end{array}$ & $\begin{array}{c}\text { Social } \\
\text { Economic }\end{array}$ & $\overline{.30}$ & $\begin{array}{l}.30 \\
-\end{array}$ & .45 & -.45 & Net & $\checkmark$ \\
\hline $\begin{array}{c}\text { Economic } \\
\text { Social }\end{array}$ & $\begin{array}{c}\text { Social } \\
\text { Economic }\end{array}$ & $\overline{.30}$ & .30 & .45 & -.20 & Net & $X$ \\
\hline $\begin{array}{c}\text { Economic } \\
\text { Social }\end{array}$ & $\begin{array}{c}\text { Social } \\
\text { Economic }\end{array}$ & $\overline{.30}$ & $\begin{array}{l}-.30 \\
-\end{array}$ & .45 & -.45 & Cooperative & $\checkmark$ \\
\hline $\begin{array}{l}\text { Economic } \\
\text { Social }\end{array}$ & $\begin{array}{c}\text { Social } \\
\text { Economic }\end{array}$ & $\overline{.30}$ & .00 & .45 & -.15 & Classical & $\checkmark$ \\
\hline
\end{tabular}

\section{The Present Investigation}

Using three community samples, we sought to identify instances of suppression for social and economic political ideology using a broad swath of theoretically relevant external criteria, including variables reflecting cognitive rigidity, authoritarianism, dangerous worldview, and lethal partisanship. We predict that statistically controlling for economic conservatism will enhance positive bivariate relations between social conservatism, on the one hand, and authoritarianism, rigidity, and dangerous worldview, on the other. We also predict that statistically controlling for social conservatism will either (a) enhance negative bivariate relations between economic conservatism and these variables (i.e., cooperative suppression) or (b) reveal negative partial regression coefficients for positive bivariate coefficients (i.e., net suppression). Replicable suppression effects, if present in our data, would suggest that the potentially divergent psychological origins of social and economic ideology can be ascertained even in samples in which social and economic conservatism manifest pronounced positive zeroorder correlations.

Further, given the extensive research literature examining relations between personality and political ideology, we examine suppressor phenomena using the HEXACO model of 
personality. The HEXACO model (Honesty-humility, Emotionality, Extraversion, Agreeableness, Conscientiousness, Openness to Experience; Ashton \& Lee, 2007) overlaps with, but differs in important ways from, the better-known five factor model (FFM; Costa \& McCrae, 1995). Specifically, unlike low FFM Agreeableness, low HEXACO Agreeableness entails ill temper, which also defines the low pole of FFM Neuroticism. Emotionality, unlike FFM Neuroticism, includes sentimentality, which also relates to FFM Agreeableness. Further, Honesty-humility has no direct parallel in the FFM, although some of Honesty-humility's defining traits are incorporated by FFM Agreeableness.

\section{Method}

\section{Participants}

In all three samples, participants were recruited using Amazon's Mechanical Turk (MTurk) platform. Research suggests that MTurk data are comparable in quality to data from other convenience samples (Buhrmester, Talaifar, \& Gosling, 2018; cf. Chmielewski \& Kucker, 2020). Still, it is necessary to screen MTurk data for errant responding. To that end, we excluded participants on the basis of failure to correctly answer two or more attention check questions. We aimed to collect approximately 500 participants in each sample. To evaluate statistical power, we implemented a Monte Carlo power analyses for indirect (i.e., suppression) effects using the procedures described in Schoemann et al. (2017). We specified 5,000 replications with 10,000 Monte Carlo draws per replication. Using population parameters of $r=.65$ for social and economic conservatism's correlation and $r=.30$ for both social and economic conservatism's relation with the $\mathrm{Y}$ variable suggested that using 500 participants ensures statistical power with a lower limit of $87 \%$ for the hypothesized indirect (i.e., suppression) effect. We also used parameters consistent with cooperative suppression (i.e., social conservatism's correlation with 
$\mathrm{Y}=.30$, economic conservatism's correlation with $\mathrm{Y}=-.20$ ), which yielded a statistical power approaching $100 \%$ for 500 participants.

\section{Sample 1}

Sample 1 comprised 489 participants $\left(\mathrm{M}_{\mathrm{age}}=41.1, \mathrm{SD}_{\mathrm{age}}=12.6\right)$. Participants were predominantly female $(53 \%)$ and white $(85.5 \%)$. The plurality were Democrats $(42.7 \%)$, with most others identifying as either Republicans $(27.0 \%)$ or independents $(24.7 \%)$. This degree of political skew is typical of online community samples (Levay, Freese, \& Druckman, 2016).

\section{Sample 2}

Sample 2 comprised 490 participants, with an average age of 38.2 years $(\mathrm{SD}=12.1)$ and a relatively even gender split (47.9\% female). Most participants identified as either Republicans (26.7\%), Democrats (47.8\%), or independents (12.9\%).

\section{Sample 3}

Sample 3 comprised 508 participants, who were $49.8 \%$ female with a mean age of 37.7 $(\mathrm{SD}=11.5)$. Most participants $(52.4 \%)$ self-identified as Democrats with a minority selfidentifying as Republicans (27.4\%) and fewer as independents (9.1\%).

\section{Measures}

All measures are reproduced, in full, in online supplemental materials.

\section{Sample 1}

Political Ideology. Political ideology was assessed using the Social and Economic Conservatism Scale (SECS; Everett, 2013), a survey of favorability toward 12 topics that are important to voters, of which 6 are economic (e.g., limited government) and 6 are social (e.g., traditional values). The SECS yields two dimensions, economic conservatism and social 
conservatism, which were positively correlated $(r=.53)$ and internally consistent $(\alpha \mathrm{s}=.94$ and .85 , respectively).

Authoritarian Parenting Values. Authoritarian child-rearing values were assessed with a 4-item forced-choice measure often used in psychology and political science (Feldman \& Stenner, 1997). The 4 items describe preferences for children who are obedient and respectful vs. free-spirited and independent $(\alpha=.67)$

Dogmatism. To assess dogmatism, we administered the DOG Scale (Altemeyer, 1996; $\alpha$ $=.92$ ), a 20-item measure of unjustified belief certainty.

Political Intolerance. To assess political intolerance, parallel right- and left-wing versions of intolerance items were administered following procedures from Crawford and Pilanski (2014). The 6 items were averaged to yield a composite political intolerance score; scores were collapsed across the left and right $(\alpha=.77)$.

Need for Cognitive Closure. Participants completed the short version of the Need for Closure Scale-Revised (NFC; Roets \& Van Hiel, 2011), a 15-item ( $\alpha=.88)$ self-report measure of aversion to ambiguity and preference for concrete information.

General Personality. We also administered the HEXACO Personality Inventory (HEXACO; Lee \& Ashton, 2018), a 100-item measure of normal-range personality that consists of 6 factors: Honesty-Humility, Emotionality, Extraversion, Agreeableness, Conscientiousness, and Openness to Experience ( $\alpha$ s ranged from .86 to .90).

\section{Sample 2}

Political Ideology. Participants indicated the degree to which they symbolically identified as left-wing vs. right-right in the economic and social domains on Likert-type items with 7 response options. Economic-social correlations were high, $r=.76$. 
State Control vs. Personal Freedom. Participants viewed an empty graph, with the poles of its X-axis labeled with preferences for left- vs. right-wing political policies and the poles of its y-axis labeled with preferences for a political system with substantial centralized state control vs. a political system that maximizes personal and political freedom and autonomy. Participants were instructed to place themselves within each graph by moving their mouse to the position that best represents their beliefs. We ventured that the resulting coordinate data could be decomposed and that the y-axis data would be useful as a non-partisan measure of authoritarianism.

Dogmatism. As in Sample 1, we administered the DOG Scale $(\alpha=.85)$. In addition to the DOG Scale, we constructed a semi-original measure of value-neutral dogmatism, adopting or slightly altering the wording of 8 items from Rokeach's (1960) Dogmatism (D) Scale and adding 10 items with good face validity for Rokeach's conceptualization of dogmatism as generalized authoritarianism ( $\alpha=.80$; see Table S4 for items and factor loadings). This modified D Scale manifested a large correlation with the DOG Scale, $r=.49$.

General personality. As in Sample 1, we administered the HEXACO Personality Inventory (Lee \& Ashton, 2018); as ranged from .86 to .90 .

\section{Sample 3}

Political Ideology. As in Sample 2, Participants indicated the degree to which they symbolically identified as left-wing vs. right-wing in the economic and social domains. Economic-social correlations were high, $r=.81$.

Authoritarian Parenting Values. As in Sample 1, authoritarian child-rearing values were assessed with a 4-item forced-choice measure (Feldman \& Stenner, 1997); $\alpha=.63$ ). 
State Control vs. Personal Freedom. The same procedures were followed as described for Sample 2.

Extreme Partisanship. Items to assess partisan moral disengagement (5-items, $\alpha=.88$ ), political violence (4-items, $\alpha=.88$ ), and partisan schadenfreude (3-items, $\alpha=.86$ ) were adapted from Kalmoe and Mason (2019). Moral disengagement items describe the rationalization of harm towards political opponents; political violence items describe lessened restraint towards killing political opponents; and partisan schadenfreude items describe lessened sympathy towards suffering of political opponents. For purposes of clarity and to avoid double-barreled items, the items were slightly modified from their original wording (e.g., the item "Would you say [Opposing party] are a serious threat to the United States and its people, or wouldn't you go that far?" was rewritten as "[Opposing party] are a serious threat to the United States and its people.")

Dangerous Worldview. Altemeyer's (1996) 12-item Belief in a Dangerous World Scale was used to assess a dangerous worldview. We modified one double-barreled item and modified another to balance potentially partisan content $(\alpha=.88)$.

\section{Mutual Suppression Analyses}

We followed an analytic approach outlined by de Zavala, Cichocka, and Bilewicz (2013) to test for mutual suppressor effects, performing a series of multiple regression analyses with social and economic political ideology as predictors and external criteria as outcomes to test our hypotheses, using bootstrapping (see Hayes, 2009) to generate point estimates, 95\% confidence intervals, and effect sizes for each potential suppressor effect (analytic code available upon request). We first regressed each outcome on either social or economic conservatism (step 1 of the regression). Next, each outcome was regressed on social and economic conservatism 
simultaneously (step 2). This process was repeated, such that if social conservatism had been entered in step 1, and economic conservatism entered in step 2, we then estimated a regression model with economic conservatism in step 1 and social conservatism in step 2 , revealing the extent to which economic and social conservatism are better predictors when controlling for one another. Any increases in size and/or changes in directionality of regression coefficients across steps 1 and 2 were observed, as were any differences in the amount of variance explained by the full model (step 2) vs. sole predictor models (step 1).

To provide more formal tests of our mutual suppression hypotheses, we used the PROCESS macro (Model 4; Hayes, 2017) in SPSS to estimate 95\% bias-corrected bootstrap confidence intervals for the indirect effects of each ideology dimension (via the other dimension) on each outcome (i.e., first a mediation model with economic conservatism as the $\mathrm{X}$ variable and social conservatism as the suppressor/mediator, and then a model with social conservatism as the $\mathrm{X}$ variable and economic conservatism as the suppressor/mediator). Although mediation and suppression are conceptually distinct, they are statistically related, and as such point and interval estimates of mediation effects can be adapted for use in tests of suppression (MacKinnon et al., 2000). More specifically, a mediational framework assumes that the magnitude of a relation between two variables will be reduced by statistically adjusting for a third variable (i.e., mediator) because the mediator is the causal path between the two variables. In cases where a suppression effect is present, however, the direct and indirect effect of the independent variable on the dependent variable will have opposite signs. Cases where the completely standardized indirect effects $\left(a b_{c s}\right)$ for social and economic conservatism are significant and display signs opposite to one another would offer evidence for mutual suppression (Tzelgov \& Henik, 1991). 
Table 2. Suppressor effects, Sample 1.

\begin{tabular}{|c|c|c|c|c|c|c|c|c|c|c|c|}
\hline \multirow[b]{2}{*}{ Outcome } & \multirow[b]{2}{*}{ Full Model } & \multirow[b]{2}{*}{$\begin{array}{c}\text { Step } 2 \\
\text { Predictor }\end{array}$} & \multirow[b]{2}{*}{$\begin{array}{c}\text { Step } 2 \\
\Delta R_{2} \\
\end{array}$} & \multicolumn{2}{|c|}{ Step 1} & \multicolumn{2}{|c|}{$\begin{array}{c}\text { Step } 2 \\
\text { (Mutual Control) } \\
\end{array}$} & \multicolumn{2}{|c|}{$\begin{array}{l}a b_{c s} \text { Effect Size } \\
\text { (Indirect effect) }\end{array}$} & \multirow[b]{2}{*}{$\begin{array}{l}\text { Co-op or Net } \\
\text { Suppression? }\end{array}$} & \multirow[b]{2}{*}{$\begin{array}{c}\text { Mutual } \\
\text { Suppression? }\end{array}$} \\
\hline & & & & $\begin{array}{c}\text { Social } \\
\beta\end{array}$ & $\begin{array}{c}\text { Economic } \\
\beta \\
\end{array}$ & $\begin{array}{c}\text { Social } \\
\beta\end{array}$ & $\begin{array}{c}\text { Economic } \\
\beta \\
\end{array}$ & PE & $95 \% \mathrm{CI}$ & & \\
\hline \multirow[t]{2}{*}{ Auth. Parenting } & \multirow{2}{*}{$\begin{array}{c}F(2,476)=70.02 \\
R_{2}=.23, p<.001\end{array}$} & Social & $.197 * *$ & - & $.17 * *$ & $.53 * *$ & $-.11 *$ & $.28 * *$ & {$[.22, .35]$} & \multirow[t]{2}{*}{ Net } & \multirow[t]{2}{*}{$\mathrm{X}$} \\
\hline & & Economic & $.008^{*}$ & $.47 * *$ & - & $.53 * *$ & $-.11 *$ & $-.06^{*}$ & {$[-.11,-.01]$} & & \\
\hline \multirow{2}{*}{$\begin{array}{c}\text { Political } \\
\text { Intolerance }\end{array}$} & \multirow{2}{*}{$\begin{array}{l}F(2,473)=6.32 \\
R 2=.03, p=.002\end{array}$} & Social & $.017 *$ & - & -.09 & $.16^{*}$ & $-.18 *$ & $.08 * *$ & {$[.03, .14]$} & \multirow[t]{2}{*}{ Cooperative } & \multirow[t]{4}{*}{$\checkmark$} \\
\hline & & Economic & $.022 * *$ & .06 & - & $.16^{*}$ & $-.18 *$ & $-.09 * *$ & {$[-.15,-.04]$} & & \\
\hline \multirow{2}{*}{$\begin{array}{l}\text { Dogmatism } \\
\text { (DOG Scale) }\end{array}$} & \multirow{2}{*}{$\begin{array}{c}F(2,476)=13.89, \\
R_{2}=.06, p<.001\end{array}$} & Social & $.036^{* *}$ & - & $.14^{*}$ & $.22 * *$ & .02 & $.12 * *$ & {$[.06, .18]$} & \multirow[t]{2}{*}{$X$} & \\
\hline & & Economic & .000 & $.23 * *$ & - & $.22 * *$ & .02 & .01 & {$[-.05, .07]$} & & \\
\hline \multirow[t]{2}{*}{ Need for Closure } & \multirow{2}{*}{$\begin{array}{l}F(2,476)=14.97 \\
R 2=.06, p<.001\end{array}$} & Social & $.054 * *$ & - & .07 & $.28 * *$ & -.08 & $.15^{* *}$ & {$[.09, .25]$} & \multirow[t]{2}{*}{ Net } & \multirow[t]{2}{*}{$\checkmark$} \\
\hline & & Economic & .004 & $.24 * *$ & - & $.28 * *$ & -.08 & -.04 & {$[-.10, .02]$} & & \\
\hline \multirow[t]{2}{*}{ Honesty-humility } & \multirow{2}{*}{$\begin{array}{l}F(2,475)=4.031 \\
R 2=.02, p=.018\end{array}$} & Social & $.014^{*}$ & - & -.05 & $.14^{*}$ & $-.13 *$ & $.07 * *$ & {$[.01, .14]$} & \multirow[t]{2}{*}{ Cooperative } & \multirow[t]{2}{*}{$\checkmark$} \\
\hline & & Economic & $.012 *$ & .07 & - & $.14 *$ & $-.13 *$ & $-.07 * *$ & {$[-.13,-.01]$} & & \\
\hline \multirow[t]{2}{*}{ Emotionality } & \multirow{2}{*}{$\begin{array}{c}F(2,475)=13.66 \\
R_{2}=.05, p<.001\end{array}$} & Social & $.030 * *$ & - & $-.16 * *$ & $.21 * *$ & $-.27 * *$ & $.11 * *$ & {$[.05, .17]$} & \multirow[t]{2}{*}{ Cooperative } & \multirow[t]{2}{*}{$\checkmark$} \\
\hline & & Economic & $.050 * *$ & .06 & - & $.21 * *$ & $-.27 * *$ & $-.14 * *$ & {$[-.20,-.08]$} & & \\
\hline Extraversion & $F(2,475)=4.63$ & Social & $.008^{*}$ & - & $.10^{*}$ & $.11 *$ & .05 & $.06^{*}$ & {$[.00, .12]$} & $X$ & \\
\hline & $R_{2}=.02, p=.010$ & Economic & .002 & $.13 *$ & - & $.11^{*}$ & .05 & .03 & {$[-.03, .08]$} & & \\
\hline Agreeableness & $F(2,475)=2.95$ & Social & $.012 *$ & - & -.02 & $.13^{*}$ & -.09 & $.07 * *$ & {$[.00, .14]$} & Cooperative & $\checkmark$ \\
\hline & $R_{2}=.01, p=.053$ & Economic & .006 & .08 & - & $.13^{*}$ & -.09 & -.05 & {$[-.11, .01]$} & & \\
\hline Conscientiousness & $F(2,475)=1.27$ & Social & .000 & - & ns & ns & ns & .04 & {$[-.03, .10]$} & $\mathrm{X}$ & \\
\hline & $R_{2}=.01, p=.283$ & Economic & .003 & ns & - & ns & ns & .00 & {$[-.06, .07]$} & & \\
\hline Openness & $F(2,475)=24.72$ & Social & $.045^{* *}$ & - & $-.22 * *$ & $-.25 * *$ & -.09 & $.13^{* *}$ & {$[-.20,-.08]$} & $X$ & \\
\hline & $R_{2}=.10, p<.001$ & Economic & .006 & $-.30 * *$ & - & $-.25 * *$ & -.09 & -.05 & {$[-.11, .01]$} & & \\
\hline
\end{tabular}

outcome before controlling for the other conservatism dimension. In Step 2, the conservatism dimensions are mutually controlled for one another. 
Table 3. Suppressor effects, Sample 2.

\begin{tabular}{|c|c|c|c|c|c|c|c|c|c|c|c|}
\hline \multirow[b]{2}{*}{ Outcome } & \multirow[b]{2}{*}{ Full Model } & \multirow[b]{2}{*}{$\begin{array}{c}\text { Step } 2 \\
\text { Predictor }\end{array}$} & \multirow[b]{2}{*}{$\begin{array}{l}\text { Step } 2 \\
\Delta R_{2}\end{array}$} & \multicolumn{2}{|c|}{ Step 1} & \multicolumn{2}{|c|}{$\begin{array}{c}\text { Step } 2 \text { (Mutual } \\
\text { Control) }\end{array}$} & \multicolumn{2}{|c|}{$a b_{c s}$ Effect Size } & \multirow[b]{2}{*}{$\begin{array}{l}\text { Co-op or Net } \\
\text { Suppression? }\end{array}$} & \multirow[b]{2}{*}{$\begin{array}{c}\text { Mutual } \\
\text { Suppression? }\end{array}$} \\
\hline & & & & $\begin{array}{c}\text { Social } \\
\beta\end{array}$ & $\begin{array}{c}\text { Economic } \\
\beta \\
\end{array}$ & $\begin{array}{c}\text { Social } \\
\beta\end{array}$ & $\begin{array}{c}\text { Economic } \\
\beta \\
\end{array}$ & $\mathrm{PE}$ & $95 \% \mathrm{CI}$ & & \\
\hline $\begin{array}{l}\text { Dogmatism } \\
\text { (DOG Scale) }\end{array}$ & $\begin{array}{l}F(2,458)=26.83 \\
R 2=.11, p<.001\end{array}$ & $\begin{array}{c}\text { Social } \\
\text { Economic }\end{array}$ & $\begin{array}{l}.082 * * \\
.014 *\end{array}$ & $30 * *$ & $\begin{array}{c}.15 * * \\
-\end{array}$ & $\begin{array}{l}.44 * * \\
.44 * *\end{array}$ & $\begin{array}{l}-.18 * \\
-.18 *\end{array}$ & $\begin{array}{l}.33 * * \\
-.14 * *\end{array}$ & $\begin{array}{c}{[.22,45]} \\
{[-.25,-.03]}\end{array}$ & Net & $\checkmark$ \\
\hline $\begin{array}{l}\text { Dogmatism } \\
\text { (D Scale) }\end{array}$ & $\begin{array}{l}F(2,454)=22.15 \\
R 2=.09, p<.001\end{array}$ & $\begin{array}{c}\text { Social } \\
\text { Economic }\end{array}$ & $\begin{array}{l}.088^{* *} \\
.043 * *\end{array}$ &.$\overline{21 * *}$ & .03 & $\begin{array}{l}.45^{* *} \\
.45^{* *}\end{array}$ & $\begin{array}{l}-.32 * * \\
-.32 * *\end{array}$ & $\begin{array}{l}.34 * * \\
-.24 * *\end{array}$ & $\begin{array}{c}{[.24, .46]} \\
{[-.36,-.14]}\end{array}$ & Net & $\checkmark$ \\
\hline State Control & $\begin{array}{l}F(2,451)=10.49 \\
R 2=.04, p<.001\end{array}$ & $\begin{array}{c}\text { Social } \\
\text { Economic }\end{array}$ & $\begin{array}{l}.019 * \\
.043 * *\end{array}$ & $\overline{-.03}$ & $\begin{array}{c}-.16 * * \\
-\end{array}$ & $\begin{array}{l}.22 * \\
.22 *\end{array}$ & $\begin{array}{l}-.32 * * \\
-.32 * *\end{array}$ & $\begin{array}{l}.17 * * \\
-.25 * *\end{array}$ & $\begin{array}{c}{[.06, .27]} \\
{[-.19,-.08]}\end{array}$ & Cooperative & $\checkmark$ \\
\hline Honesty-humility & $\begin{array}{l}F(2,459)=1.54 \\
R_{2}=.01, p=.216\end{array}$ & $\begin{array}{c}\text { Social } \\
\text { Economic }\end{array}$ & $\begin{array}{l}\mathrm{ns} \\
\mathrm{ns}\end{array}$ & $\overline{\mathrm{ns}}$ & $\underline{\mathrm{ns}}$ & $\begin{array}{l}\mathrm{ns} \\
\mathrm{ns}\end{array}$ & $\begin{array}{l}\mathrm{ns} \\
\mathrm{ns}\end{array}$ & $\begin{array}{l}\mathrm{ns} \\
\mathrm{ns}\end{array}$ & $\begin{array}{l}\mathrm{ns} \\
\mathrm{ns}\end{array}$ & $\mathrm{X}$ & \\
\hline Emotionality & $\begin{array}{l}F(2,459)=10.47 \\
R 2=.04, p<.001\end{array}$ & $\begin{array}{c}\text { Social } \\
\text { Economic }\end{array}$ & $\begin{array}{l}.000 \\
.018^{*}\end{array}$ & $-\overline{16 * *}$ & $-.21 * *$ & $\begin{array}{l}-.00 \\
-.00\end{array}$ & $\begin{array}{l}-.21 * * \\
-.21 * *\end{array}$ & $\begin{array}{c}.00 \\
-.16^{* *}\end{array}$ & $\begin{array}{l}{[-.11, .10]} \\
{[-.27,-.04]}\end{array}$ & $\mathrm{X}$ & \\
\hline Extraversion & $\begin{array}{l}F(2,458)=2.60 \\
R_{2}=.01, p=.075\end{array}$ & $\begin{array}{c}\text { Social } \\
\text { Economic }\end{array}$ & $\begin{array}{l}\mathrm{ns} \\
\mathrm{ns}\end{array}$ & - & ns & $\begin{array}{l}\mathrm{ns} \\
\mathrm{ns}\end{array}$ & $\begin{array}{l}\mathrm{ns} \\
\mathrm{ns}\end{array}$ & $\begin{array}{l}\mathrm{ns} \\
\mathrm{ns}\end{array}$ & $\begin{array}{l}\mathrm{ns} \\
\mathrm{ns}\end{array}$ & $\mathrm{X}$ & \\
\hline Agreeableness & $\begin{array}{l}F(2,459)=0.19 \\
R_{2}=.00, p=.981\end{array}$ & $\begin{array}{c}\text { Social } \\
\text { Economic }\end{array}$ & $\begin{array}{l}\mathrm{ns} \\
\mathrm{ns}\end{array}$ & $\overline{\mathrm{ns}}$ & $\underline{\mathrm{ns}}$ & $\begin{array}{l}\mathrm{ns} \\
\mathrm{ns}\end{array}$ & $\begin{array}{l}\mathrm{ns} \\
\mathrm{ns}\end{array}$ & $\begin{array}{l}\mathrm{ns} \\
\mathrm{ns}\end{array}$ & $\begin{array}{l}\mathrm{ns} \\
\mathrm{ns}\end{array}$ & $\mathrm{X}$ & \\
\hline Conscientiousness & $\begin{array}{l}F(2,459)=3.82 \\
R_{2}=.02, p=.023\end{array}$ & $\begin{array}{c}\text { Social } \\
\text { Economic }\end{array}$ & $\begin{array}{l}.002 \\
.012 *\end{array}$ & $\overline{.06}$ & $\begin{array}{l}.12 \\
-\end{array}$ & $\begin{array}{l}-.06 \\
-.06\end{array}$ & $\begin{array}{l}.17^{*} \\
.17^{*}\end{array}$ & $\begin{array}{l}-.05 \\
.13 * *\end{array}$ & $\begin{array}{c}{[-.18, .06]} \\
{[.02, .25]}\end{array}$ & Net & $X$ \\
\hline Openness & $\begin{array}{c}F(2,458)=31.20 \\
R_{2}=.12, p<.001\end{array}$ & $\begin{array}{c}\text { Social } \\
\text { Economic }\end{array}$ & $\begin{array}{l}.030 * * \\
.004\end{array}$ & $-\overline{34 * *}$ & $-.30 * *$ & $\begin{array}{l}-.27 * * \\
-.27 * *\end{array}$ & $\begin{array}{l}-.10 \\
-.10\end{array}$ & $\begin{array}{l}-.20 * * \\
-.08\end{array}$ & $\begin{array}{c}{[-.32,-.09]} \\
{[-.18, .03]}\end{array}$ & $X$ & \\
\hline
\end{tabular}

Note. $*$ indicates $p<.05 ; * *$ indicates $p<.001$. Step 1 and Step 2 refer to the regression model. Step 1 describes the relation between social or economic conservatism and each outcome before controlling for the other conservatism dimension. In Step 2, the conservatism dimensions are mutually controlled for one another. 
Table 4. Suppressor effects, Sample 3.

\begin{tabular}{|c|c|c|c|c|c|c|c|c|c|c|c|}
\hline \multirow[b]{2}{*}{ Outcome } & \multirow[b]{2}{*}{ Full Model } & \multirow[b]{2}{*}{$\begin{array}{c}\text { Step } 2 \\
\text { Predictor }\end{array}$} & \multirow[b]{2}{*}{$\begin{array}{c}\text { Step } 2 \\
\Delta R_{2} \\
\end{array}$} & \multicolumn{2}{|c|}{ Step 1} & \multicolumn{2}{|c|}{$\begin{array}{c}\text { Step } 2 \text { (Mutual } \\
\text { Control) }\end{array}$} & \multicolumn{2}{|c|}{$a b_{c s}$ Effect Size } & \multirow[b]{2}{*}{$\begin{array}{l}\text { Co-op or Net } \\
\text { Suppression? }\end{array}$} & \multirow[b]{2}{*}{$\begin{array}{c}\text { Mutual } \\
\text { Suppression? }\end{array}$} \\
\hline & & & & $\begin{array}{c}\text { Social } \\
\beta \\
\end{array}$ & $\begin{array}{c}\text { Economic } \\
\beta \\
\end{array}$ & $\begin{array}{c}\text { Social } \\
\beta\end{array}$ & $\begin{array}{c}\text { Economic } \\
\beta \\
\end{array}$ & $\mathrm{PE}$ & $95 \% \mathrm{CI}$ & & \\
\hline Auth. Parenting & $\begin{array}{l}F(2,501)=79.18 \\
R_{2}=.24, p<.001\end{array}$ & $\begin{array}{c}\text { Social } \\
\text { Economic }\end{array}$ & $\begin{array}{l}.096 * * \\
.001\end{array}$ & $\overline{.49 * *}$ & $\begin{array}{c}.38 * * \\
-\end{array}$ & $\begin{array}{l}.53 * * \\
.53 * *\end{array}$ & $\begin{array}{l}-.05 \\
-.05\end{array}$ & $\begin{array}{l}.43^{* *} \\
-.04\end{array}$ & $\begin{array}{l}{[.33, .53]} \\
{[-.14, .06]}\end{array}$ & Net & $\mathrm{X}$ \\
\hline State Control & $\begin{array}{l}F(2,505)=32.32, \\
R_{2}=.11, p<.001\end{array}$ & $\begin{array}{c}\text { Social } \\
\text { Economic }\end{array}$ & $\begin{array}{c}.004 \\
.059 * *\end{array}$ & $-.23 * *$ & $\begin{array}{c}-.33 * * \\
-\end{array}$ & $\begin{array}{l}.10 \\
.10\end{array}$ & $\begin{array}{l}-.42 * * \\
-.42 * *\end{array}$ & $\begin{array}{c}.08 \\
-.34 * *\end{array}$ & $\begin{array}{l}{[-.01, .19]} \\
{[-.45,-.23]}\end{array}$ & Net & $X$ \\
\hline $\begin{array}{c}\text { Moral } \\
\text { Disinhibition }\end{array}$ & $\begin{array}{l}F(2,503)=4.03 \\
R_{2}=.02, p=.018\end{array}$ & $\begin{array}{c}\text { Social } \\
\text { Economic }\end{array}$ & $\begin{array}{l}.012^{*} \\
.016^{*}\end{array}$ & $\overline{.01}$ & $\begin{array}{l}-.06 \\
-\end{array}$ & $\begin{array}{l}.19 * * \\
.19 * *\end{array}$ & $\begin{array}{l}-.22 * \\
-.22 *\end{array}$ & $\begin{array}{l}.15 * * \\
-.17 * *\end{array}$ & $\begin{array}{c}{[.04, .28]} \\
{[-.30,-.06]}\end{array}$ & Cooperative & $\checkmark$ \\
\hline Political Violence & $\begin{array}{l}F(2,504)=4.65 \\
R_{2}=.02, p=.010\end{array}$ & $\begin{array}{c}\text { Social } \\
\text { Economic }\end{array}$ & $\begin{array}{l}.013^{*} \\
.003\end{array}$ & $\overline{.12 *}$ & $\begin{array}{l}.07 \\
-\end{array}$ & $\begin{array}{l}.20 * \\
.20 *\end{array}$ & $\begin{array}{l}-.09 \\
-.09\end{array}$ & $\begin{array}{l}.16^{* *} \\
-.08\end{array}$ & $\begin{array}{l}{[.05, .29]} \\
{[-.19, .04]}\end{array}$ & Net & $\checkmark$ \\
\hline $\begin{array}{c}\text { Partisan } \\
\text { Schadenfreude }\end{array}$ & $\begin{array}{l}F(2,505)=7.82, \\
R_{2}=.03, p<.001\end{array}$ & $\begin{array}{c}\text { Social } \\
\text { Economic }\end{array}$ & $\begin{array}{l}.001 \\
.016^{*}\end{array}$ & $-\overline{12 *}$ & $\begin{array}{c}-.17 * * \\
-\end{array}$ & $\begin{array}{l}.06 \\
.06\end{array}$ & $\begin{array}{l}-.22 * \\
-.22 *\end{array}$ & $\begin{array}{c}.05 \\
-.18 * *\end{array}$ & $\begin{array}{l}{[-.07, .17]} \\
{[-.29,-.06]}\end{array}$ & Net & $\mathrm{X}$ \\
\hline $\begin{array}{l}\text { Dangerous } \\
\text { Worldview }\end{array}$ & $\begin{array}{l}F(2,500)=14.64 \\
R_{2}=.06, p<.001\end{array}$ & $\begin{array}{c}\text { Social } \\
\text { Economic }\end{array}$ & $\begin{array}{l}.042 * * \\
.010 *\end{array}$ & $-\overline{.21 *}$ & $.12 * *$ & $\begin{array}{l}.35 * * \\
.35 * *\end{array}$ & $\begin{array}{l}-.17 * \\
-.17 *\end{array}$ & $\begin{array}{l}.28 * * \\
-.14 * *\end{array}$ & $\begin{array}{c}{[.17, .40]} \\
{[-.25,-.02]}\end{array}$ & Net & $\checkmark$ \\
\hline $\begin{array}{l}\text { Dogmatism } \\
\text { (D Scale) }\end{array}$ & $\begin{array}{l}F(2,491)=27.54, \\
R_{2}=.13, p<.001\end{array}$ & $\begin{array}{c}\text { Social } \\
\text { Economic }\end{array}$ & $\begin{array}{c}.064 * * \\
.003 \\
\end{array}$ &.$\overline{36^{* *}}$ & $\begin{array}{c}.26^{* *} \\
- \\
\end{array}$ & $\begin{array}{l}.43^{* *} \\
.43^{* *} \\
\end{array}$ & $\begin{array}{l}-.09 \\
-.09 \\
\end{array}$ & $\begin{array}{c}-.35 * * \\
.07 \\
\end{array}$ & $\begin{array}{l}{[-.49,-.22]} \\
{[-.05, .20]}\end{array}$ & Net & $X$ \\
\hline
\end{tabular}

Note. * indicates $p<.05 ; *$ indicates $p<.001$. Step 1 and Step 2 refer to the regression model. Step 1 describes the relation between social or economic conservatism and each

outcome before controlling for the other conservatism dimension. In Step 2, the conservatism dimensions are mutually controlled for one another. 


\section{Results}

Results1 are presented in Tables 2, 3, and 4 (unstandardized suppressor effect sizes are presented in supplemental materials).

\section{Sample 1}

Of the variables that we predicted would yield cooperative or net suppressor effects, 3 of 4 demonstrated significant suppressor effects for social and/or economic conservatism. Namely, suppression was present for authoritarian parenting, political intolerance, and need for closure, whereas little evidence of suppression emerged for the DOG Scale. In the case of authoritarianism, controlling for social conservatism resulted in the initially positive relation between economic conservatism and authoritarianism becoming negative, with a significant suppression effect $\left(a b_{c s}=.28,95 \%\right.$ CI $\left.[.22, .35]\right)$, while controlling for economic conservatism enhanced the positive relation between social conservatism and authoritarianism, with a significant suppression effect $\left(a b_{c s}=-.06,95 \%\right.$ CI $\left.[-.11,-.01]\right)$. Similarly, controlling for social conservatism resulted in the initially positive relation between economic conservatism and need for closure becoming negative, $a b_{c s}=.15,95 \% \mathrm{CI}(.09, .25)$, while controlling for economic conservatism enhanced the positive relation between social conservatism and need for closure, albeit not significantly, $a b_{c s}=-.04,95 \% \mathrm{CI}(-.10, .02)$. Hence, net suppression was present for both authoritarianism and need for closure. Mutual suppression, however, was present only in the case of need for closure, as the magnitude of economic conservatism's partial coefficient was not larger than its corresponding bivariate coefficient (Nickerson, 2008).

1 Data will be available in a public repository on the Open Science Framework upon publication. 
Social conservatism was initially positively related to political intolerance, whereas economic conservatism was initially negatively related to political intolerance. After controlling for economic conservatism, the positive relation for social conservatism became larger, $a b_{c s}=-$ $.09,95 \%$ CI (-.15, -.04). Similarly, after controlling for social conservatism, the negative relation for economic conservatism became larger, $a b_{c s}=.08,95 \% \mathrm{CI}(.03, .14)$. Thus, cooperative suppression emerged for political intolerance.

Our exploratory analyses with personality also yielded several significant suppressor effects. Namely, honesty-humility, emotionality, and agreeableness manifested cooperative suppressor effects. In all three cases, social conservatism's initially positive relation became larger after controlling for economic conservatism ( $a b_{c s}$ ranged from -.14 to -.05), whereas economic conservatism's initially negative relation became larger after controlling for social conservatism ( $a b_{c s}$ ranged from .07 to .11).

\section{Sample 2}

In the second sample, all three hypothesized suppressor effects were present. For both measures of dogmatism, initially positive relations with economic conservatism became negative and increased in magnitude after controlling for social conservatism (DOG Scale $a b_{c s}=.33,95 \%$ CI $[.22, .45]$; D Scale $a b_{c s}=.34,95 \%$ CI $\left.[.24, .46]\right)$. Similarly, initially positive relations between social conservatism and dogmatism increased after controlling for economic conservatism (DOG Scale $a b_{c s}=-.14,95 \%$ CI [-.25, -.03]; D Scale $a b_{c s}=-.24,95 \%$ CI [-.36, .14]). Concerning preferences for state control, an initially negative relation with social conservatism became positive and larger in magnitude after controlling for economic conservatism, $a b_{c s}=-.25,95 \% \mathrm{CI}(-.19,-.08)$, and an initially negative relation with economic 
conservatism became larger in magnitude after controlling for social conservatism, $a b_{c s}=.17$, 95\% CI (.06, .27). Thus, mutual suppression emerged for all three outcome variables.

The HEXACO cooperative suppression effects found in Sample 1 did not replicate in Sample 2. Also in contrast with results from Sample 1, a net suppression effect was present for conscientiousness, such that an initial positive relation between conscientiousness and social conservatism became negative, with a significant suppressor effect $\left(a b_{c s}=.13,95 \% \mathrm{CI}[.02\right.$, $.25]$ ), whereas an initial positive relation between conscientiousness and economic conservatism became larger, but the suppressor effect was non-significant $\left(a b_{c s}=-.05,95 \%\right.$ CI $\left.[-.18, .06]\right)$.

\section{Sample 3}

Of the six hypothesized suppressor effects in Sample 3, all six were present for either social and/or economic conservatism. Still, only three instances of mutual suppression emerged. For moral disinhibition, net suppression was apparent, such that the initially negative relation with social conservatism became positive and larger in magnitude after controlling for economic conservatism, $a b_{c s}=-.17,95 \% \mathrm{CI}(-.30,-.06)$, while the negative relation with economic conservatism became larger after controlling for social conservatism, $a b_{c s}=.15,95 \% \mathrm{CI}(.04$, .28). Similarly, for dangerous worldview, social conservatism's positive relation became larger after controlling for economic conservatism, $a b_{c s}=-.14,95 \% \mathrm{CI}(-.25,-.02)$, and economic conservatism's positive relation became negative and larger in magnitude after controlling for social conservatism, $a b_{c s}=.28,95 \% \mathrm{CI}(.17, .40)$. Both cases reflect mutual suppression.

For political violence, social conservatism's positive coefficient became larger after economic control, but the suppression effect was not significant, $a b_{c s}=-.08,95 \% \mathrm{CI}(-.19, .04)$; in contrast, economic conservatism's positive relation became negative and larger in magnitude after social control, with a significant suppression effect $\left(a b_{c s}=.16,95 \% \mathrm{CI}[.05, .29]\right)$. 
Concerning partisan schadenfreude, social conservatism's negative relation became positive, but not larger, after controlling for economic conservatism, with a significant suppression effect $\left(a b_{c s}\right.$ $=-.18,95 \%$ CI $[-.29,-.06])$, while economic conservatism's negative relation became larger after controlling for social conservatism, but the suppression effect was not significant, $a b_{c s}=.05$, $95 \%$ CI $(-.07, .17)$.

Replicating the finding of net suppression in Sample 1, controlling for social conservatism resulted in the initially positive relation between economic conservatism and authoritarianism becoming negative, but not larger in magnitude, with a significant suppression effect, $a b_{c s}=.43,95 \% \mathrm{CI}(.33, .53)$, whereas economic control increased the positive coefficient of social conservatism, but the suppression effect was not significant, $a b_{c s}=-.04,95 \% \mathrm{CI}(-.14$, .06). Replicating our findings in Sample 2, a negative relation between state control preferences and social conservatism became positive, but not larger in magnitude, after controlling for economic conservatism, with a significant suppression effect, $a b_{c s}=-.34,95 \% \mathrm{CI}(-.45,-.23)$. The significant negative relation between preferences for state control and economic conservatism became larger after controlling for social conservatism, but the suppression effect was non-significant, $a b_{c s}=.08,95 \% \mathrm{CI}(-.01, .19)$. Also replicating Sample 2's results, net suppression was present for the D Scale, such that the significant positive relation between economic conservatism became negative, but not larger, after controlling for social conservatism, $a b_{c s}=-.25,95 \% \mathrm{CI}(-.19,-.08)$. The positive relation between the D Scale and social conservatism became larger after controlling for economic conservatism, but the suppressor effect was non-significant, $a b_{c s}=-.07,95 \% \mathrm{CI}(-.20, .05)$.

\section{Discussion}


Across three samples, economic and social ideology acted as mutual suppressors for the outcomes of political intolerance, need for closure, dogmatism, preferences for state control, moral disinhibition, political violence, and dangerous worldview. Non-mutual net suppression was present in the case of authoritarian parenting and partisan schadenfreude. Taken together, these findings suggest that social and economic conservatism differ in their psychological implications (Carmines et al., 2012; Feldman \& Johnston, 2014).

Consistent with observations that left-wing economic preferences and right-wing social preferences are undergirded by shared psychological mechanisms, our research revealed that economic conservatism often becomes negatively related to psychological needs for certainty and safety after controlling for shared nonspecific variance in social conservatism, while social conservatism's relations with these variables increases. These results may challenge the sufficiency of theories linking psychological mechanisms to support for a broad-based political conservatism (e.g., Jost et al., 2003), but also suggest that social conservatism's relations with various external criteria may often be underestimated in the literature.

Effect sizes were particularly large for authoritarian child-rearing values and support for state control, corroborating Malka and colleagues' (2017) speculation that motivations for governmental protection (vs. motivations for individual freedoms) may lead people to adopt culturally right-wing attitudes and economically left-wing attitudes (see also Lefkofridi et al., 2014). Historical examples of left- vs. right-wing authoritarianism also support this possibility. Highly repressive left-wing regimes in the 20 th and $21_{\text {st }}$ century, such as those in the Soviet Union, China, East Germany, Cambodia, and Cuba tended to emphasize communism, a primarily economic political philosophy, whereas right-wing authoritarian states, such as Italy, Spain, Nazi Germany, and Chile tended to emphasize social unity, strength, and purity by 
exalting a nation or race above all else (Paxton, 2004). One can also cite several authoritarian regimes in which left-wing economic elements and right-wing social elements were closely intermingled, such as Libya, Syria, Iraq, and Iran.

Our findings demonstrate that "purer" indicators of social conservatism and economic conservatism are more strongly related to criterion variables than might otherwise be reported. To that end, and on a more practical note, we recommend that researchers studying the causes and correlates of political ideology measure both social and economic ideology and attempt to statistically distinguish social from economic ideology following the procedures outlined in this investigation. Even in cases where mutual suppression is not present, disentangling social from economic ideology may be informative. For instance, although regressing trait openness on social and economic ideology did not enhance the strength of either predictor, in our doing so it became clear that social conservatism drives the negative relation between openness and global conservatism. Given that openness-conservatism relations are among the most replicated findings in political and personality psychology (Carney et al., 2008; Chirumbolo \& Leone, 2010; Mondak, 2010), the possibility that this relation is largely or entirely attributable to social conservatism may have far-reaching implications for the overlap between personality and politics.

The heterogeneity of measures of political ideology should be carefully considered in interpreting our results. In Sample 1, in which we measured social and economic political ideology by way of political issue preferences, the social-economic correlation was approximately $r=.50$, whereas when we assessed political ideology via symbolic identification, correlations were closer to $r=.80$. Notwithstanding the magnifying effects of these high correlations on any potential suppressor situations, Zell and Bornstein (2014) found evidence of 
disparities between political ideologies as measured by single-item self-placement relative to participants' issue preferences; participants tended to perceive themselves as more conservative than they actually were. Measures that assess substantive policy preferences, rather than political affiliation, may be better suited to capturing variation across social and economic ideology. As such our use of single-item measures is a major limitation of findings drawn from Samples 2 and 3.

The present studies entirely relied on self-report measures, rather than laboratory-based or behavioral measures, raising the specter of mono-method bias. Future research examining social-economic suppressor phenomena in external criteria measured via observer-reports, behavioral measures, and/or meta-cognitive tasks would provide more robust corroboration of our findings. Another limitation is our reliance on MTurk samples, which tend to be more liberal, educated, and politically engaged than those from the general population. Our findings might differ in a representative or right-skewed sample. For instance, given that the general population is less likely to be politically engaged than are MTurkers, the magnitude of social-economic correlations, along with the effect sizes for suppression effects, may be smaller in nationally representative data. Replication of mutual suppressor effects in more ideologically diverse samples is therefore needed.

\section{Conclusion}

The abstract ideas comprising the left-right political spectrum are not entirely logically or psychologically coherent (Converse, 1964). Accordingly, whether one espouses "left-wing” or "right-wing" political preferences may be as much a function of tribal animus, sacred cultural values, and social media, talk radio, and cable television pushing people to adopt simplistic rather than nuanced positions on many political issues as it is of one's psychological features. At 
the very least, there are statistically unique elements of social and economic conservatism that demonstrate opposing patterns of relations with psychological variables. Moreover, per the present research, to the extent that social and economic ideology are organically structured due to epistemic commonalities, free-market conservatism may dovetail more with social liberalism than with social conservatism. 


\section{References}

Adorno, T. W., Frenkel-Brunswik, E., Levinson, D. J., \& Sanford, R. N. (1950). The authoritarian personality. New York: Harper.

Altemeyer, R. A., \& Altemeyer, B. (1996). The authoritarian specter. Harvard University Press.

Ashton, M. C., \& Lee, K. (2007). Empirical, theoretical, and practical advantages of the HEXACO model of personality structure. Personality and Social Psychology Review, 11, $150-166$.

Azevedo, F., Jost, J. T., Rothmund, T., \& Sterling, J. (2019). Neoliberal ideology and the justification of inequality in capitalist societies: Why social and economic dimensions of ideology are intertwined. Journal of Social Issues, 75, 49-88.

Caprara, G. V., \& Vecchione, M. (2018). On the left and right ideological divide: Historical accounts and contemporary perspectives. Political Psychology, 39, 49-83.

Carl, N. (2014). Verbal intelligence is correlated with socially and economically liberal beliefs. Intelligence, 44, 142-148.

Carmines, E. G., Ensley, M. J., \& Wagner, M. W. (2012, October). Political ideology in American politics: one, two, or none? In The Forum (Vol. 10, No. 3). De Gruyter.

Carney, D. R., Jost, J. T., Gosling, S. D., \& Potter, J. (2008). The secret lives of liberals and conservatives: Personality profiles, interaction styles, and the things they leave behind. Political Psychology, 29, 807-840.

Cizmar, A. M., Layman, G. C., McTague, J., Pearson-Merkowitz, S., \& Spivey, M. (2014). Authoritarianism and American political behavior from 1952 to 2008. Political Research Quarterly, 67, 71-83. 
Conger, A. J. (1974). A revised definition for suppressor variables: A guide to their identification and interpretation. Educational and Psychological Measurement, 34, 35-46.

Cohen, J. \& Cohen, P. (1975). Applied multiple correlation/regression analysis for the social sciences. New York: Wiley.

Converse P. (1964). Ideology and discontent. New York: The Free Press.

Cornelis, I., \& Van Hiel, A. (2006). The impact of cognitive styles on authoritarianism based conservatism and racism. Basic and Applied Social Psychology, 28, 37-50.

Costa Jr, P. T., \& McCrae, R. R. (1995). Domains and facets: Hierarchical personality assessment using the Revised NEO Personality Inventory. Journal of personality assessment, 64, 21-50.

Costello, T.H. (in press). The Conundrum of Measuring Authoritarianism: A Case Study in Political Bias. In W. O’Donohue \& C. Frisby (Eds.), Political bias in psychology. New York, NY: Springer.

Costello, T.H., Bowes, S.M., Lilienfeld, S.O. (2020). "Escape from Freedom": Authoritarianismrelated traits, political ideology, personality, and belief in free will/determinism. Journal of Research in Personality, 86, 1-16.

Costello, T.H., Bowes, S.M., Malka, A., Baldwin, M., Lilienfeld, S.O. (Manuscript under review). Thinking, left and right: The rigidity-of-the-right revisited.

Crawford, J. T., \& Pilanski, J. M. (2014). Political intolerance, right and left. Political Psychology, 35, 841-851.

de Zavala, A. G., Cichocka, A., \& Bilewicz, M. (2013). The paradox of in-group love: Differentiating collective narcissism advances understanding of the relationship between in-group and out-group attitudes. Journal of Personality, 81, 16-28. 
Duckitt, J., \& Sibley, C. G. (2009). A dual-process motivational model of ideology, politics, and prejudice. Psychological Inquiry, 20, 98-109.

Everett, J. A. (2013). The 12 item social and economic conservatism scale (SECS). PloS one, 8, e82131.

Federico, C. M. (2020). When do psychological differences predict political differences? Engagement and the psychological bases of political polarization. In Political Polarization. Routledge.

Federico, C. M., \& Schneider, M. C. (2007). Political expertise and the use of ideology: Moderating effects of evaluative motivation. Public Opinion Quarterly, 71, 221-252.

Feldman, S., \& Johnston, C. (2014). Understanding the determinants of political ideology: Implications of structural complexity. Political Psychology, 35, 337-358.

Feldman, S., \& Stenner, K. (1997). Perceived threat and authoritarianism. Political Psychology, 18, 741-770.

Goren, P. (2013). On voter competence. Oxford University Press.

Hayes, A. F. (2009). Beyond Baron and Kenny: Statistical mediation analysis in the new millennium. Communication Monographs, 76, 408-420.

Hibbing, J. R., Smith, K. B., \& Alford, J. R. (2014). Differences in negativity bias underlie variations in political ideology. Behavioral and Brain Sciences, 37, 297-350.

Hetherington, M., \& Weiler, J. (2018). Prius Or Pickup?: How the Answers to Four Simple Questions Explain America's Great Divide. Houghton Mifflin.

Iyer, R., Koleva, S., Graham, J., Ditto, P., \& Haidt, J. (2012). Understanding libertarian morality: The psychological dispositions of self-identified libertarians. PloS one, 7. 
Johnson, S. D., \& Tamney, J. B. (2001). Social traditionalism and economic conservatism: Two conservative political ideologies in the United States. The Journal of Social Psychology, 141, 233-243.

Johnston, C. D., \& Ollerenshaw, T. (2020). How different are cultural and economic ideology? Current Opinion in Behavioral Sciences, 34, 94-101.

Johnston, C. D., \& Wronski, J. (2015). Personality dispositions and political preferences across hard and easy issues. Political Psychology, 36, 35-53.

Jost, J. T. (2017). Ideological asymmetries and the essence of political psychology. Political Psychology, 38, 167-208.

Jost, J. T., Glaser, J., Kruglanski, A. W., \& Sulloway, F. J. (2003). Political conservatism as motivated social cognition. Psychological Bulletin, 129, 339-375.

Jost, J. T., Federico, C. M., \& Napier, J. L. (2013). Political ideologies and their social psychological functions. The Oxford handbook of political ideologies.

Kalmoe, N. P. (2020). Uses and abuses of ideology in political psychology. Political Psychology, 41, 771-793.

Kalmoe, N. P., \& Mason, L. (2019). Lethal mass partisanship: Prevalence, correlates, and electoral contingencies. In NCAPSA American Politics Meeting.

Laméris, M. D., Jong-A-Pin, R., \& Garretsen, H. (2018). On the measurement of voter ideology. European Journal of Political Economy, 55, 417-432.

Lakens, D. (2014, December 19). Observed power, and what to do if your editor asks for posthoc power analyses [Web log post]. Retrieved from http://daniellakens.blogspot.com/2014/12/observed-power-and-what-to-do-ifyour.html. 
Lilienfeld, S. O., Watts, A. L., Murphy, B., Costello, T. H., Bowes, S. M., Smith, S. F., ... \& Tabb, K. (2019). Personality disorders as emergent interpersonal syndromes: Psychopathic personality as a case example. Journal of Personality Disorders, 33, 577622.

Lee, K., \& Ashton, M. C. (2018). Psychometric properties of the HEXACO-100. Assessment, 25, 543-556.

Lee, K., Ashton, M. C., Griep, Y., \& Edmonds, M. (2018). Personality, religion, and politics: An investigation in 33 countries. European Journal of Personality, 32, 100-115.

Lefkofridi, Z., Wagner, M., \& Willmann, J. E. (2014). Left-authoritarians and policy representation in Western Europe: Electoral choice across ideological dimensions. West European Politics, 37, 65-90.

Levay, K. E., Freese, J., \& Druckman, J. N. (2016). The demographic and political composition of Mechanical Turk samples. Sage Open, 6, 2158244016636433.

MacKinnon, D. P., Krull, J. L., \& Lockwood, C. M. (2000). Equivalence of the mediation, confounding and suppression effect. Prevention Science, 1, 173-181.

Malka, A., Lelkes, Y., \& Soto, C. J. (2017). Are cultural and economic conservatism positively correlated? A large-scale cross-national test. British Journal of Political Science, 49, 1045-1069.

Malka, A., \& Soto, C. J. (2015). Rigidity of the economic right? Menu-independent and menudependent influences of psychological dispositions on political attitudes. Current Directions in Psychological Science, 24, 137-142. 
Malka, A., Soto, C. J., Inzlicht, M., \& Lelkes, Y. (2014). Do needs for security and certainty predict cultural and economic conservatism? A cross-national analysis. Journal of Personality and Social Psychology, 106, 1031-1051.

Malka, A., \& Lelkes, Y. (2010). More than ideology: Conservative-liberal identity and receptivity to political cues. Social Justice Research, 23, 156-188.

Morris, M. W., Carranza, E., \& Fox, C. R. (2008). Mistaken identity: Activating conservative political identities induces "conservative" financial decisions. Psychological Science, 19, 1154-1160.

Nickerson, C. (2008). Mutual Suppression: Comment on Paulhus et al. (2004). Multivariate Behavioral Research, 43, 556-563.

Orth, U., Robins, R. W., Meier, L. L., \& Conger, R. D. (2016). Refining the vulnerability model of low self-esteem and depression: Disentangling the effects of genuine self-esteem and narcissism. Journal of Personality and Social Psychology, 110, 133-149.

Orth, U., Berking, M., \& Burkhardt, S. (2006). Self-conscious emotions and depression: Rumination explains why shame but not guilt is maladaptive. Personality and Social Psychology Bulletin, 32, 1608-1619.

Pan, J., \& Xu, Y. (2018). China's ideological spectrum. The Journal of Politics, 80, 254-273.

Paulhus, D. L., Robins, R. W., Trzesniewski, K. H., \& Tracy, J. L. (2004). Two replicable suppressor situations in personality research. Multivariate Behavioral Research, 39(2), 303-328.

Paxton, R. O. (2007). The anatomy of fascism. Vintage. 
Preacher, K. J., \& Hayes, A. F. (2004). SPSS and SAS procedures for estimating indirect effects in simple mediation models. Behavior Research Methods, Instruments, \& Computers, 36, 717-731.

Rokeach, M. (1960). The open and closed mind: investigations into the nature of belief systems and personality systems. Basic Books.

Schoemann, A. M., Boulton, A. J., \& Short, S. D. (2017). Determining power and sample size for simple and complex mediation models. Social Psychological and Personality Science, 8, 379-386.

Sterling, J., Jost, J. T., \& Pennycook, G. (2016). Are neoliberals more susceptible to bullshit? Judgment \& Decision Making, 11, 352-360.

Tracy, J. L., \& Robins, R. W. (2006). Appraisal antecedents of shame and guilt: Support for a theoretical model. Personality and Social Psychology Bulletin, 32, 1339-1351.

Tracy, J. L., Cheng, J. T., Robins, R. W., \& Trzesniewski, K. H. (2009). Authentic and hubristic pride: The affective core of self-esteem and narcissism. Self and Identity, 8, 196-213.

Tzelgov, J., \& Henik, A. (1991). Suppression situations in psychological research: Definitions, implications, and applications. Psychological Bulletin, 109, 524-536.

Van Hiel, A., Pandelaere, M., \& Duriez, B. (2004). The impact of need for closure on conservative beliefs and racism: Differential mediation by authoritarian submission and authoritarian dominance. Personality and Social Psychology Bulletin, 30, 824-837.

Vitriol, J. A., Larsen, E. G., \& Ludeke, S. G. (2019). The generalizability of personality effects in politics. European Journal of Personality. Pagination forthcoming. 
Watson, D., Clark, L. A., Chmielewski, M., \& Kotov, R. (2013). The value of suppressor effects in explicating the construct validity of symptom measures. Psychological Assessment, 25, 929.

Wiggins, J. S. (1973). Personality and prediction: Principles of personality assessment. Addison-Wesley Pub. Co

Yı1maz, O., Sarıbay, S. A., Bahçekapıl1, H. G., \& Harma, M. (2016). Political orientations, ideological self-categorizations, party preferences, and moral foundations of young Turkish voters. Turkish Studies, 17, 544-566.

Zaller, J. R. (1992). The nature and origins of mass opinion. Cambridge University Press.

Zell, E., \& Bernstein, M. J. (2014). You may think you're right... Young adults are more liberal than they realize. Social Psychological and Personality Science, 5, 326-333. 